\title{
Improved low crosstalk, high transmission and quality factor eight channel demultiplexer based on photonic crystal
}

Mohammad Azadi

Shahid Rajaee Teacher Training University

Masoud Mohammadi

Shahid Rajaee Teacher Training University

Saeed Olyaee

Shahid Rajaee Teacher Training University

mahmood seifouri ( $\triangle$ mahmood.seifouri@sru.ac.ir)

Shahid Rajaee Teacher Training University

\section{Research Article}

Keywords: All-optical, demultiplexers, crosstalk, transmission and quality factor

Posted Date: May 10th, 2022

DOl: https://doi.org/10.21203/rs.3.rs-1297878/v2

License: (a) (i) This work is licensed under a Creative Commons Attribution 4.0 International License.

Read Full License 


\section{Abstract}

Due to the importance of all-optical demultiplexers (DEMUXs) in optical communication networks, this study aims to design and simulate a novel type of DEMUX using 2D photonic crystals (2D-PhCs). The proposed structure consists of one input waveguide and eight output channels considering linear and point defects. To design this DEMUX, silicon dielectric rods with the refractive index of 3.45 are employed

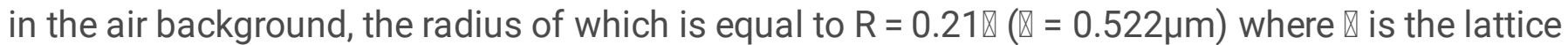
constant. The photonic band gap (PBG) and output spectrum of the structure are analyzed by the plane wave expansion (PWE) and finite-difference time-domain (FDTD) methods, respectively. The simulation results indicate the average quality factor and transmission efficiency are 3,602 and $98 \%$, respectively, and inter-channel crosstalk ranges from -60.5 to $-25.5 \mathrm{~dB}$.

\section{Introduction}

Fiber-optic communication systems transmit data with lower attenuation over long distances than radio frequency (RF) communication systems )Azadi et al. 2021; Mohammadi and Seifouri 2018(. Recent advances in modern technologies and high speed of light are among the important factors influencing the development of integrated optical devices. This approach gradually leads to the replacement of classical electronic systems with next-generation optical systems. In optical communication networks, light waves transmit data over very long distances with excellent speed (Balaji et al. 2020; Mohammadi et al. 2020; Manzacca et al. 2006; Mohammadi and Seifouri 2019). In these networks, light waves travel inside optical fibers which are designed in the infrared frequency range at the wavelength of $0.8-1.65 \mu \mathrm{m}$ and transmit data across the ultra-wideband of about $1 \mathrm{THz}$ (Zahedi et al. 2019; Balaji et al. 2017; Mohammadi et al. 2021; Fallahi and Seifouri 2018).

Optical networks become easier and cheaper when they are routed through SMF for a large number of users. Wavelength division multiplexing (WDM) technology along with SMF is applied to send a number of unique light waves through the same SMF. At the receiver's end, the light emitted from the SMF is distributed to the user separately by DEMUX (Mohammadi and Seifouri 2019; Alipour-Banaei et al. 2015; Naghizade and Mohammadi 2020; Ghezali et al. 2018; Fallahi et al. 2019).

To construct ultra-compact optical DEMUXs, the structures should be able to control the propagation of light waves inside very small space and waveguides (Moradi et al. 2020; Rostami et al. 2011; AlipourBanaei et al. 2013). Due to their small size, excellent performance in optical sensors, high-speed data processing and optical networks, photonic crystals (PhCs) have provided new opportunities for designing all-optical devices required for realizing all the integrated optical circuits, communication networks and data processing systems (Rajarajan et al. 2016; Babu and Sreenivasulu 2021; Balaji et al. 2021; Absalan 2018). Photonic crystals are composed of alternating layers of insulating materials. PBG, as one of their unique properties (Delphi et al. 2019; Masilamani and Punniakodi 2020; Mehdizadeh et al. 2016), is defined as a specific frequency (or wavelength) range, in which the propagation of electromagnetic waves is prohibited in any direction and can be controlled by different structural parameters such as 
lattice constant, dielectric constant of the material and rod radius (Mohammadi et al. 2021; Zhuang et al. 2016; Mansouri-Birjandi and Rakhshani 2013; Ahmed et al. 2013). Electromagnetic waves propagate inside the PBG when a defect is formed.

There are usually two types of defects, namely linear and point, for PBG. Linear defect occurs by removing or changing structural parameters (lattice constant, refractive index and rod radius) of the entire row of rods in the PhC structure. Point defect occurs by completely removing or changing structural parameters of the rod. Designing PhC structures requires the introduction of one or both types of defects (Veisi et al. 2021; Jindal and Kaur 2018; Saral et al. 2016; Dharchana et al. 2017; Gupta and Medhekar 2014). These structures are applied in various optical devices such as design and fabrication of optical waveguides, optical lenses, optical filters, optical MUXs/DEMUXs, optical splitters, photonic sensors, optical converters, all-optical logic gates, optical modulators/demodulators and other types of optical devices. Various PhC-based devices have been proposed so far (Danaie et al. 2018; Mahmoud et al. 2004; Haq Shaik and Rangaswamy 2017; Rezaee et al. 2015). Significant advances in the field of PhCs have raised hopes for having more all-optical systems and replacing electrons with photons (Liu et al. 2020; Moumeni and Labbani 2021).

PhC-based DEMUXs play a key role in WDM techniques and can be used as the basic block in realizing some other optical digital devices (Singh and Singh 2017; Talebzadeh et al. 2020; Sreenivasulu et al. 2018). Hence, different methods have been presented for designing PhC-based optical DEMUXs. Nowadays, PhC-based DEMUXs have received great attention from researchers due to their intrinsic properties such as low energy consumption, very small size, low loss, better performance, etc. (Robinson and Nakkeeran 2013; Granpayeh et al. 2018; Bernier et al. 2008). The number of output channels, transmission efficiency, quality factor, crosstalk and inter-channel spacing are among the most important properties of PhC-based DEMUXs (Zhang et al. 2012; Boumami and Naoum 2014). Most of the studies have been conducted on 4-channel DEMUXs. The efficiency of DEMUX technologies depends on the number of optical channels existing inside the optical fiber. To increase the number of channels inside the optical fiber, it is necessary to design optical DEMUXs considering more output channels. Therefore, designing 8-channel DEMUXs is more useful than 4-channel DEMUXs.

Various types of 8-channel optical DEMUXs have been designed by researchers so far, which have provided acceptable results in the third telecommunication window. Alipour et al. (2013) designed a PhCbased 8-channel DEMUX and achieved the average quality factor of 2,955, maximum crosswalk of $8 \mathrm{~dB}$ and transmission efficiency of 56\% (Alipour-Banaei et al. 2013). Mehdizadeh et al. (2016) presented an 8channel DEMUX with PhC-based ring nano-resonator and reported the maximum quality factor, transmission efficiency and maximum inter-channel crosstalk as 2,200, 94-99\% and $-11.2 \mathrm{~dB}$, respectively (Mehdizadeh and Soroosh 2016). Venkatachkam et al. (2016) proposed a PhC-based 8channel DEMUX and achieved the quality factor of 825 and transmission efficiency of $80 \%$ (Venkatachalam et al. 2017). Talebzadeh et al. presented PhC-based 6- and 8-channel DEMUXs. The lowest transmission efficiency and quality factor as well as the highest inter-channel crosstalk of the 8channel DEMUX were obtained as $91 \%, 3,716$ and $-11 \mathrm{~dB}$, respectively (Talebzadeh et al. 2017). 
Moharrami et al. (2019) designed an 8-channel DEMUX based on a photonic crystal with dimensions of $18 \times 77$. The average quality factor and transmission coefficient of this structure were obtained as 1,319 and $98 \%$, respectively. The crosstalk ranged from -40 to $-16 \mathrm{~dB}$ (Mohammadi et al. 2019). Rakhshani (2020) presented an 8-channel DEMUX based on 2D-PhCs and reported the quality factor, transmission coefficient and average inter-channel crosstalk of 1,000, $96 \%$ and $-35 \mathrm{~dB}$, respectively (Rakhshani 2020). Reviewing the reported DEMUXs, this paper aims to address the shortcomings of previous structures such as transmission efficiency, quality factor and inter-channel crosstalk. Therefore, this study presents a novel structure of PhC-based DEMUX. The rest of this paper is organized as follows:

Section 2 examines PBG using PWE method and presents the final structure of the proposed DEMUX. Section 3 presents the simulation results using FDTD method and compares results of this work with those o previous studies. Section 4 provides the conclusion.

\section{Proposed Demux}

This study aims to present a PhC-based 8-channel DEMUX. For this purpose, the $63 \times 31$ array of dielectric rods was used in $X$ and $Z$ directions in the air background. The structure lengths were $33 \mu \mathrm{m}$ and $16 \mu \mathrm{m}$ in $X$ and $Z$ directions, respectively. The area of the proposed structure was considered to be $528 \mu \mathrm{m}^{2}$.

The design of $\mathrm{PhC}$ structures depends on parameters such as lattice constant $(\nabla)$, radius of dielectric rods $(R)$, refractive index of dielectric rods ( $n$ ) and material of dielectric rods. The desired PBG could be

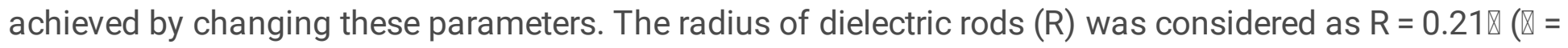
$0.522 \mu \mathrm{m})$, where $\otimes$ is the lattice constant, i.e., all the four dielectric rods were located at square corners, the side length of which was equal to $0.522 \mu \mathrm{m}$. The dielectric rods were made of silicon with the refractive index of $3.45\left(n_{\mathrm{si}}=3.45\right)$. Important structural parameters illustrated in Table 1 (all radii were normalized to the lattice constant, $\mathbb{\otimes})$.

Table 1

Important structural parameters.

\begin{tabular}{|llll|}
\hline $\mathrm{n}_{\mathrm{si}}$ & $\mathrm{n}_{\text {air }}$ & $\mathbb{}$ & $\mathbf{R}$ \\
\hline 3.45 & 1 & $522 \mathrm{~nm}$ & $0.21 \rrbracket$ \\
\hline
\end{tabular}

The structure should have the appropriate PBG within the desired wavelength range in order to conduct light waves in photonic crystals. For this purpose, PBG was calculated by PWE method. The PWE method follows Maxwell's equations, and calculates TE and TM band gaps as follows (Balaji et al. 2017; Talebzadeh et al. 2017):

$\Delta \times\left(\frac{1}{\varepsilon(r)} \Delta \times E(r)\right)=\frac{\omega^{2}}{c^{2}} E(r)[1]$

where $\varepsilon(r)$ is the dielectric constant, $E(r)$ is the frequency-dependent electric field vector, $\omega$ is the angular frequency and $\mathrm{c}$ is the light speed in vacuum. Figure 1 indicates PBG diagram of the proposed structure. 
The PBG was in the range of $1.29 \mu \mathrm{m}<\lambda<1.86 \mu \mathrm{m}$, which was suitable for optical communications in the third telecommunication window.

Choosing the structure wavelength was the most important part in designing the PhC-based DEMUX. The input waveguide was created by removing 55 dielectric rods from the central row of the structure. Then, four output waveguides were created both at the top and bottom of the structure by removing and changing the radius of dielectric rods. At each output, several dielectric rods were removed and the radius of the dielectric rod was changed. Using different radii for the eight outputs provided different wavelengths for them. Table 2 presents the defect rod radius related to the proposed DEMUX structure. Figure 2 indicates the final structure of the proposed DEMUX.

Table 2

Defect rod radius (rod/区)

\begin{tabular}{|c|c|c|c|c|c|c|c|c|}
\hline & Ch1 & Ch2 & Ch3 & Ch4 & Ch5 & Ch6 & Ch7 & Ch8 \\
\hline $\mathrm{R}_{1} / \mathbb{\Delta}(\mathrm{nm})$ & 105 & 105 & 106 & 106 & 107 & 107 & 108 & 108 \\
\hline $\mathrm{R}_{2} / \mathbb{\Delta ( n m )}$ & 142 & 148 & 149 & 150 & 156 & 152 & 152 & 156 \\
\hline
\end{tabular}

\section{Simulation Results}

The simulation was performed using FDTD method in order to investigate the optical behavior of the proposed DEMUX and the output spectrum was plotted in terms of wavelength. To use this method, accurate meshing and time calculations were required. The following equation was applied (Delphi et al. 2019):

$\Delta t \leq \frac{1}{c \sqrt{\left(\frac{1}{\Delta \mathrm{x}^{2}}-\frac{1}{\Delta \mathrm{z}^{2}}\right)}}[2]$

where $\Delta \mathrm{x}$ and $\Delta \mathrm{z}$ denote mesh sizes of the structure, $\Delta \mathrm{t}$ represents time steps of the structure and $\mathrm{c}$ is the light speed in open space.

The 8-channel DEMUX is a key process in data transmission. In this study, the main goal was to design an optical DEMUX with 8 output channels at very low bandwidth in the third telecommunication window due to low losses. The lattice constant $(\mathbb{\nabla})$ had to be changed in order to place the output channels in the range of $1.55 \mu \mathrm{m}$ in the proposed structure. The output spectrum of the structure tended toward lower wavelengths by reducing the lattice constant, so that the output spectrum of the structure was in the wavelength range of C-band at the lattice constant of $0.522 \mu \mathrm{m}$. Then, the appropriate output spectrum was obtained for the eight output channels in terms of quality factor, transmission efficiency and crosstalk by managing the refractive index and radius of dielectric rods of the structure and performing the trial and error of defect rods in the output path. 
Figure 3 illustrates the output spectrum of the proposed DEMUX, which included 8 channels with the central wavelengths of $\lambda_{1}=1.5271 \mu \mathrm{m}, \lambda_{2}=1.5337 \mu \mathrm{m}, \lambda_{3}=1.5395 \mu \mathrm{m}, \lambda_{4}=1.5446 \mu \mathrm{m}, \lambda_{5}=1.55 \mu \mathrm{m}, \lambda_{6}$ $=1.5542 \mu \mathrm{m}, \lambda_{7}=1.5587 \mu \mathrm{m}$ and $\lambda_{8}=1.5632 \mu \mathrm{m}$, all of which were in the C-band telecommunication window.

As shown in Fig. 3, the average overall efficiency and channel spacing were $98 \%$ and $5 \mathrm{~nm}$, respectively.

Quality factor (QF) is another important parameter in DEMUX. The quality factor of DEMUX for each channel was calculated as follows:

$\mathrm{Q}=\frac{\lambda_{0}}{\Delta \lambda}[3]$

where QF is the quality factor, $\lambda_{0}$ is the central wavelength and $\Delta \lambda$ is the channel bandwidth. According to the above equation, lower bandwidth was associated with a higher quality factor. Therefore, in the proposed structure, the minimum and maximum quality factors were 2,739 and 4,277, respectively. Table 3 presents details of the output spectrum of each channel such as central wavelength, bandwidth, quality factor and transmission efficiency.

Table 3

The output characteristics of the eight PhC DEMUX

\begin{tabular}{|lllllllll|}
\hline & Ch1 & Ch2 & Ch3 & Ch4 & Ch5 & Ch6 & Ch7 & Ch8 \\
\hline $\begin{array}{l}\text { Central wavelength } \\
(\mu \mathrm{m})\end{array}$ & 1.5271 & 1.5337 & 1.5395 & 1.5446 & 1.55 & 1.5542 & 1.5587 & 1.5632 \\
\hline Band width $(\mathrm{nm})$ & 0.36 & 0.56 & 0.36 & 0.44 & 0.52 & 0.48 & 0.42 & 0.38 \\
\hline Transmission & $96 \%$ & $98 \%$ & $99 \%$ & $98 \%$ & $98.5 \%$ & $95.5 \%$ & $98 \%$ & $99.5 \%$ \\
\hline Quality Factor & 4242 & 2739 & 4277 & 3511 & 2981 & 3238 & 3712 & 4114 \\
\hline
\end{tabular}

Figure 4 (a-h) indicates the electric field distribution of the proposed DEMUX in channels $1-8$, respectively. All the input signals reached channel 1 at $\lambda_{1}=1.5271 \mu \mathrm{m}$. Therefore, the signal power of channel 1 reached the maximum value at the desired wavelength. Similarly, outputs of channels $2,3,4,5$, 6,7 and 8 received the highest input signals at $\lambda_{2}=1.5337 \mu \mathrm{m}, \lambda_{3}=1.5395 \mu \mathrm{m}, \lambda_{4}=1.5446 \mu \mathrm{m}, \lambda_{5}=1.55$ $\mu \mathrm{m}, \lambda_{6}=1.5542 \mu \mathrm{m}, \lambda_{7}=1.5587 \mu \mathrm{m}$ and $\lambda_{8}=1.5632 \mu \mathrm{m}$.

The degree of interference between adjacent channels (crosstalk) is another important indicator for evaluating the performance of the PhC-based DEMUX. The effect of crosstalk on optical communications is serious because the signal sent to a channel can create an adverse effect on another channel. In designing DEMUXs, there is less effort to achieve crosstalk. According to the output spectrum of the structure (dB) shown in Fig. 5, Table 4 presents the crosstalk. The inter-channel crosstalk ranged from 60.5 to $-25.5 \mathrm{~dB}$, which was very lower than previous similar works. 
Table 4

Inter-channel crosstalk of the 8 outputs of the proposed DEMUX

\begin{tabular}{|lllllllll|}
\hline Channel & Ch1 & Ch2 & Ch3 & Ch4 & Ch5 & Ch6 & Ch7 & Ch8 \\
\hline Ch1 & - & $-33.3 \mathrm{~dB}$ & $-48 \mathrm{~dB}$ & $-45.2 \mathrm{~dB}$ & $-37 \mathrm{~dB}$ & $-52 \mathrm{~dB}$ & $-59 \mathrm{~dB}$ & $-56 \mathrm{~dB}$ \\
\hline Ch2 & $-26.6 \mathrm{~dB}$ & - & $-30 \mathrm{~dB}$ & $-39.5 \mathrm{~dB}$ & $-30 \mathrm{~dB}$ & $-46 \mathrm{~dB}$ & $-51.5 \mathrm{~dB}$ & $-51 \mathrm{~dB}$ \\
\hline Ch3 & $-39 \mathrm{~dB}$ & $-37.5 \mathrm{~dB}$ & - & $-33 \mathrm{~dB}$ & $-34.6 \mathrm{~dB}$ & $-60.5 \mathrm{~dB}$ & $-44.5 \mathrm{~dB}$ & $-47 \mathrm{~dB}$ \\
\hline Ch4 & $-46 \mathrm{~dB}$ & $-36 \mathrm{~dB}$ & $-28.5 \mathrm{~dB}$ & - & $-32.5 \mathrm{~dB}$ & $-44 \mathrm{~dB}$ & $-45 \mathrm{~dB}$ & $-42 \mathrm{~dB}$ \\
\hline Ch5 & $-48.5 \mathrm{~dB}$ & $-40 \mathrm{~dB}$ & $-34 \mathrm{~dB}$ & $-28.5 \mathrm{~dB}$ & - & $-31.5 \mathrm{~dB}$ & $-40.5 \mathrm{~dB}$ & $-42.5 \mathrm{~dB}$ \\
\hline Ch6 & $-44.6 \mathrm{~dB}$ & $-40 \mathrm{~dB}$ & $-42.5 \mathrm{~dB}$ & $-40.4 \mathrm{~dB}$ & $-25.5 \mathrm{~dB}$ & - & $-33 \mathrm{~dB}$ & $-46.6 \mathrm{~dB}$ \\
\hline Ch7 & $-47 \mathrm{~dB}$ & $-49.8 \mathrm{~dB}$ & $-41.7 \mathrm{~dB}$ & $-49 \mathrm{~dB}$ & $-30.4 \mathrm{~dB}$ & $-30.5 \mathrm{~dB}$ & - & $-41.5 \mathrm{~dB}$ \\
\hline Ch8 & $-50 \mathrm{~dB}$ & $-54 \mathrm{~dB}$ & $-51 \mathrm{~dB}$ & $-39.7 \mathrm{~dB}$ & $-32.4 \mathrm{~dB}$ & $-39.2 \mathrm{~dB}$ & $-30 \mathrm{~dB}$ & - \\
\hline
\end{tabular}

Table 5 compares the obtained results with those of previous works in order to value the present paper. The proposed structure had higher quality factor and transmission efficiency as well as lower crosstalk than previous works. 
Table 5

Comparing results of this paper with similar previous works

\begin{tabular}{|c|c|c|c|c|c|c|}
\hline Reference & $\begin{array}{l}\text { Number } \\
\text { of } \\
\text { channels }\end{array}$ & $\begin{array}{l}\text { Channel } \\
\text { spacing (nm) }\end{array}$ & $\begin{array}{l}\text { Spectral } \\
\text { width(nm) }\end{array}$ & $\begin{array}{l}\text { Quality } \\
\text { Factor }\end{array}$ & $\begin{array}{l}\text { Transmission } \\
\text { (\%) }\end{array}$ & $\begin{array}{l}\text { Crosstalk } \\
\text { (dB) }\end{array}$ \\
\hline [2] & 3 & 4.2 & 0.32 & 4936 & 96.5 & $\begin{array}{l}-18.6 \text { up } \\
\text { to }-34.6\end{array}$ \\
\hline [4] & 4 & - & 0.217 & 7358.5 & 99.25 & $\begin{array}{l}-9.79 \text { up } \\
\text { to }-46.68\end{array}$ \\
\hline [10] & 4 & 2.75 & 0.4 & 4164 & 95.5 & $\begin{array}{l}-10.5 \text { up } \\
\text { to }-36.5\end{array}$ \\
\hline [15] & 8 & 4 & 1.2 & 1577.7 & 94.5 & $\begin{array}{l}-8 \text { up to } \\
-48.3\end{array}$ \\
\hline [26] & 5 & - & 0.22 & 6236 & 95.98 & $\begin{array}{l}-18.04 \text { up } \\
\text { to }-50.2\end{array}$ \\
\hline [49] & 8 & - & 0.65 & 2955 & 56 & $\begin{array}{l}-8 \text { up to } \\
-40\end{array}$ \\
\hline [50] & 8 & 2.1 & 0.67 & 2300 & $94-99$ & $\begin{array}{l}-11.2 \text { up } \\
\text { to }-40\end{array}$ \\
\hline [51] & 8 & 4.2 & 1.8 & 825 & 80 & - \\
\hline [52] & 8 & - & 0.59 & 4320 & 93 & $\begin{array}{l}-11 \text { up to } \\
-46\end{array}$ \\
\hline [53] & 8 & 3 & 1.2 & 1319 & 98 & $\begin{array}{l}-16 \text { up to } \\
-40\end{array}$ \\
\hline [54] & 8 & 2.25 & 1.5 & 1000 & 96 & $\begin{array}{l}-35 \text { up to } \\
-160\end{array}$ \\
\hline [56] & 4 & 2 & 0.825 & 1943 & 95.8 & $\begin{array}{l}-14 \text { up to } \\
-25.5\end{array}$ \\
\hline $\begin{array}{l}\text { Proposed } \\
\text { DEMUX }\end{array}$ & 8 & 5 & 0.44 & 3602 & 98 & $\begin{array}{l}-25.5 \text { up } \\
\text { to }-60.5\end{array}$ \\
\hline
\end{tabular}

\section{Conclusion}

This study designed and simulated an 8-channel DEMUX based on 2D-PhCs with a square structure using linear and point defects. This structure consisted of one input waveguide and eight output waveguides. The wavelength of the output channels was in the range of the third telecommunication window by setting the lattice constant. The appropriate output spectrum was obtained in terms of quality factor, transmission efficiency and crosstalk via managing the refractive index and radius of dielectric rods of the structure, and changing the radius of defect rods in the path of the output channels. One of the 
important properties of our proposed structure was that a very regular output spectrum could be achieved without moving the rods, which was very effective in the manufacturing process. The transmission efficiency, inter-channel spacing, bandwidth and quality factor of the proposed DEMUX were $98 \%, 5 \mathrm{~nm}$, $0.44 \mathrm{~nm}$ and 3,602. Moreover, inter-channel crosstalk ranged from - 60.5 to $-25.5 \mathrm{~dB}$, which improved compared to previous similar works. The functional properties of the proposed DEMUX could meet the expectations of CWDM systems. Furthermore, the area of the proposed structure was about $528 \mu \mathrm{m}^{2}$, which was very small. Therefore, it could be implemented for photonic integrated circuits.

\section{References}

1. Azadi, M., Seifouri, M., Olyaee, S.: Low crosstalk four-channel photonic crystal demultiplexer. Sadhana 46(173), (2021)

2. Mohammadi, M., Seifouri, M.: Numerical simulation of all optical demultiplexer based on pillar photonic crystal ring resonators. Int. J. Numer. Model. 32(2), 1-11 (2018)

3. Balaji, V.R., Murugan, M., Robinson, S., Hegde, G.: Design and Analysis of Waveguide Taper for Photonic Crystal Demultiplexer. Silicon 13, 3777-3793 (2020)

4. Mohammadi, M., Fallahi, V., Seifouri, M.: Ultracompact all-optical full adders using an interference effect based on 2D photonic crystal nanoring resonators. J. Comput. Electron. 20(1), 409-418 (2020)

5. Manzacca, G., Paciotti, D., Marchese, A., Moreolo, M.S., Cincotti, G.: 2D photonic crystal cavity-based WDM multiplexer. Photon Nanostruct. Fundam Appl. 5(4), 233-236 (2006)

6. Mohammadi, M., Seifouri, M.: A new proposal for a high-performance 4-channel demultiplexer based on $2 \mathrm{D}$ photonic crystal using three cascaded ring resonators for applications in advanced optical systems. Opt. Quant. Electron. 51(11), 1-15 (2019)

7. Zahedi, A., Parandin, F., Karkhanehchi, M.M., Shams, H.H., Rajamand, S.: Design and simulation of optical 4-channel demultiplexer using photonic crystals. J. Opt. Commun. 40(1), 17-20 (2019)

8. Balaji, V.R., Murugan, M., Robinson, S., Nakkeeran, R.: Design and optimization of photonic crystal based eight channel dense wavelength division multiplexing demultiplexer using conjugate radiant neural network. Opt. Quant. Electron. 49(198), (2017)

9. Mohammadi, M., Moradiani, F., Olyaee, S., Seifouri, M.: The design and 3D simulation of a new highspeed half adder based on graphene resonators. Opt. Laser Technol. 142(35), 1072-1080 (2021)

10. Fallahi, V., Seifouri, M.: A new design of a 4-channel optical demultiplexer based on photonic crystal ring resonator using a modified Y-branch. Optica Appl. 48(2), 191-200 (2018)

11. Mohammadi, M., Seifouri, M.: Numerical investigation of photonic crystal ring resonators coupled bus waveguide as a highly sensitive platform. Photon Nanostruct. Fundam Appl. 34, 11-18 (2019)

12. Alipour-Banaei, H., Serajmohammadi, S., Mehdizadeh, F.: Optical wavelength demultiplexer based on photonic crystal ring resonators. Photon Netw. Commun. 29, 146-150 (2015) 
13. Naghizade, S., Mohammadi, S.: Optical four-channel demultiplexer based on air-bridge structure and graphite-type ring resonators. Photon Netw. Commun. 40(1), 40-48 (2020)

14. Ghezali, S., Tayeboun, F., Meradi, K.A.: Nine channels wavelength division demultiplexer based upon two dimensional photonic crystal. Progress In Electromagnetics Research. 69, 107-114 (2018)

15. Fallahi, V., Mohammadi, M., Seifouri, M.: Design of Two 8-Channel Optical Demultiplexers Using 2D Photonic Crystal Homogeneous Ring Resonators. Fiber. Int. Opt. <backgroundcolor:\#FFCC66;bu>38</background-color:\#FFCC66;bu>(5), 271-284 (2019)

16. Moradi, M., Mohammadi, M., Olyaee, S., Seifouri, M.: Design and Simulation of a Fast All-Optical Modulator Based on Photonic Crystal Using Ring Resonators. Silicon 1-7 (2020)

17. Rostami, A., Banaei, H.A., Nazari, F., Bahrami, A.: An ultra compact photonic crystal wavelength division demultiplexer using resonance cavities in a modified Y-branch structure. Opt. Int. J. Light Electron. Opt. 122(16), 1481-1485 (2011)

18. Alipour-Banaei, H., Mehdizadeh, F., Serajmohammadi, S.: A novel 4-channel demultiplexer based on photonic crystal ring resonators. Optik 124(23), 5964-5967 (2013)

19. Balaji, V.R., Murugan, M., Robinson, S.: Two-dimensional photonic crystal assisted DWDM demultiplexer with uniform channel spacing. Dig. J. Nanomater Biostruct 11(4), 1125-1134 (2016)

20. Babu, D.L., Sreenivasulu, T.: 4-Channel DWDM demultiplexer on silicon photonic crystal slab. Sadhana 46(1), 1-4 (2021)

21. Balaji, V.R., Murugan, M., Robinson, S., Hegde, G.: A Novel Hybrid Channel DWDM Demultiplexer Using Two Dimensional Photonic Crystals Meeting ITU Standards. Silicon 1-12 (2021)

22. Absalan, H.: A four-channel optical demultiplexer using photonic crystal-based resonant cavities. J. Opt. Commun. 39(4), 369-373 (2018)

23. Delphi, G., Olyaee, S., Seifouri, M., Mohebzadeh-Bahabady, A.: Design of an add filter and a 2-channel optical demultiplexer with high quality factor based on nano-ring resonator. J. Comput. Electron. 18(3), 1372-1378 (2019)

24. Masilamani, S., Punniakodi, S.: Photonic crystal ring resonator based optical MUX/DEMUX design structures: a survey and comparison study. J. Opt. 49, 168-177 (2020)

25. Mehdizadeh, F., Soroosh, M., Alipour-Banaei, H.: An optical demultiplexer based on photonic crystal ring resonators. Optik Int. J. Light Electron. Opt. 127(20), 8706-8709 (2016)

26. Mohammadi, M., Seifouri, M., Olyaee, S., Karamirad, M.: Optimization and realization all-optical compact five-channel demultiplexer using 2D photonic crystal based hexagonal cavities. J. Comput. Electron. 20(2), 984-992 (2021)

27. Zhuang, Y., Ji, K., Zhou, W., Chen, H.: Design of a DWDM multi/demultiplexer based on 2-D photonic crystals. IEEE Photon. Technol. Lett. 28(15), 1669-1672 (2016)

28. Mansouri-Birjandi, M.A., Rakhshani, M.R.: A new design of tunable four port wavelength demultiplexer by photonic crystals ring resonators. Optik 124, 5923-5926 (2013) 
29. Ahmed, R., Khan, M.M., Aoni, R.A., Ahad, A.: Design, simulation \& optimization of 2D photonic crystal power splitter. Opt. Photon J. 3(02), 13-19 (2013)

30. Veisi, E., Seifouri, M., Olyaee, S.: A novel design of all-optical high speed and ultra-compact photonic crystal and logic gate based on the kerr effect. Appl. Phys. B. 127(70), (2021)

31. Jindal, P., Kaur, H.J.: Different Configurations of Photonic Crystal Power Splitters for PIC's-A Review. Int. J. Appl. Eng. Res. 13(11), 9500-9508 (2018)

32. Saral, T.B., Robinson, S., Arunkumar, R.: Two-Dimensional Photonic Crystal Based Compact Power Splitters. Int. J. Photon Optical Technol. 2(4), 1-5 (2016)

33. Dharchana, T., Sivanantharaja, A., Selvendran, S.: Design of Pressure Sensor Using 2D Photonic Crystal. Adv. Nat. Appl. Sci. 11(7), 26-30 (2017)

34. Gupta, M.M., Medhekar, S.: A versatile optical junction using photonic band-gap guidance and self collimation. Appl. Phys. Lett. 105(13), 131104-131104.4 (2014)

35. Danaie, M., Nasiri Far, R., Dideban, A.: Design of a high-bandwidth Y-shaped photonic crystal power splitter for TE modes. Int. J. Opt. Photon 12(1), 33-42 (2018)

36. Mahmoud, M.Y., Bassou, G., Metehri, F.: Channel drop filter using photonic crystal ring resonators for CWDM communication systems. Optik 125(2), 4718-4721 (2004)

37. Haq Shaik, E., Rangaswamy, N.: Multi-mode interference-based photonic crystal logic gates with simple structure and improved contrast ratio. Photon Netw. Commun. 34(1), 140-148 (2017)

38. Rezaee, S., Zavvari, M., Alipour-Banaei, H.: A novel optical filter based on H-shape photonic crystal ring resonators. Optik Int. J. Light Electron. Opt. 126(20), 2535-2538 (2015)

39. Liu, D., Citrin, D.S., Hu, S.: Compact high-performance polarization beam splitter based on a silicon photonic crystal heterojunction. Opt. Mater. 109, (2020)

40. Moumeni, I., Labbani, A.: Very high efficient of $1 \times 2,1 \times 4$ and $1 \times 8 \mathrm{Y}$ beam splitters based on photonic crystal ring slot cavity. Opt. Quant. Electron. 53(129), (2021)

41. Singh, S., Singh, K.: Design of an integrated multi-arm power splitter using photonic crystal waveguide. Optik 145, 495-502 (2017)

42. Talebzadeh, R., Mehdizadeharhad, F., Naseri, A.: 4-channel tunable optical demultiplexer based on nonlinearity phenomenon in 2D resonant cavity photonic crystals. Frequenz 74(1-2), 9-15 (2020)

43. Sreenivasulu, T., Kaustav, B., Shafeek, A.S., Yadunath, T.R., Badrinarayana, T., Gopalkrishna, H., Srinivas, T.: Photonic crystal based four channel DWDM de-multiplexer on SOI platform: design and analysis. Opt. Eng. 57(4), (2018)

44. Robinson, S., Nakkeeran, R.: Photonic crystal ring resonator-based add drop filters: a review. Opt. Eng. 52(6), (2013)

45. Granpayeh, A., Habibiyan, H., Parvin, P.: Photonic crystal directional coupler for all-optical switching, tunable multi/demultiplexing and beam splitting applications. J. Mod. Opt. 66(4), 1-8 (2018)

46. Bernier, D., Le Roux, X., Lupu, A., Marris-Morini, D., Vivien, L., Cassan, E.: Compact, low cross-talk CWDM demultiplexer using photonic crystal superprism. Opt. Express 16(22), 17209-17214 (2008) 
47. Zhang, X., Liao, Q., Yu, T., Liu, N., Huang, Y.: Novel ultra compact wavelength division demultiplexer based on photonic band gap. Opt. Commun. 285(3), 274-276 (2012)

48. Boumami, S., Naoum, R.: New version of seven wavelengths demultiplexer based on the microcavities in a two-dimensional photonic crystal. Optik Int. J. Light Electron. Opt. 125(23), 70727074 (2014)

49. Alipour-Banaei, H., Mehdizadeh, F., Hassangholizadeh-Kashtiban, M.: A novel proposal for all optical PhC-based demultiplexers suitable for DWDM applications. Opt. Quant. Electron. 45(10), 1063-1075 (2013)

50. Mehdizadeh, F., Soroosh, M.: A new proposal for eight-channel optical demultiplexer based on photonic crystal resonant cavities. Photon Netw. Commun. 31, 65-70 (2016)

51. Venkatachalam, K., Sriram Kumar, D., Robinson, S.: Investigation on 2D photonic crystal-based eightchannel wavelength-division demultiplexer. Photon Netw. Commun. 34, 100-110 (2017)

52. Talebzadeh, R., Soroosh, M., Kavian, Y.S., Mehdizadeh, F.: All-optical 6- and 8-channel demultiplexers based on photonic crystal multilayer ring resonators in Si/C rods. Photon Netw. Commun. 34, 248257 (2017)

53. Mohammadi, B., Soroosh, M., Kovsarian, A., Seifi Kavian, Y.: Improving the transmission efficiency in eight-channel all optical Demultiplexers. Photon Netw. Commun. 38(1), 115-120 (2019)

54. Rakhshani, M.R.: Compact eight-channel wavelength demultiplexer using modified photonic crystal ring resonators for CWDM applications. Photon Netw. Commun. 39, 143-151 (2020)

55. Delphi, Gh.A., Olyaee, S., Seifouri, M.: Software-based teaching of optical demultiplexers by using photonic crystals with presenting the syllabus for MSc students in electronic engineering major. Tech. Edu J. 13(4), 762-774 (2019)

56. Viswanathan, B., Rajesh, A., Jeyalakshmi, C., Abinaya, S., Deepika, J., Dharini, D., Gajasree, S.: Design of 6-channel optical demultiplexer/coupler using photonic crystal for IFoF in $5 \mathrm{G}$ networks. Opt. Quant. Electron. 53(444), (2021)

\section{Figures}




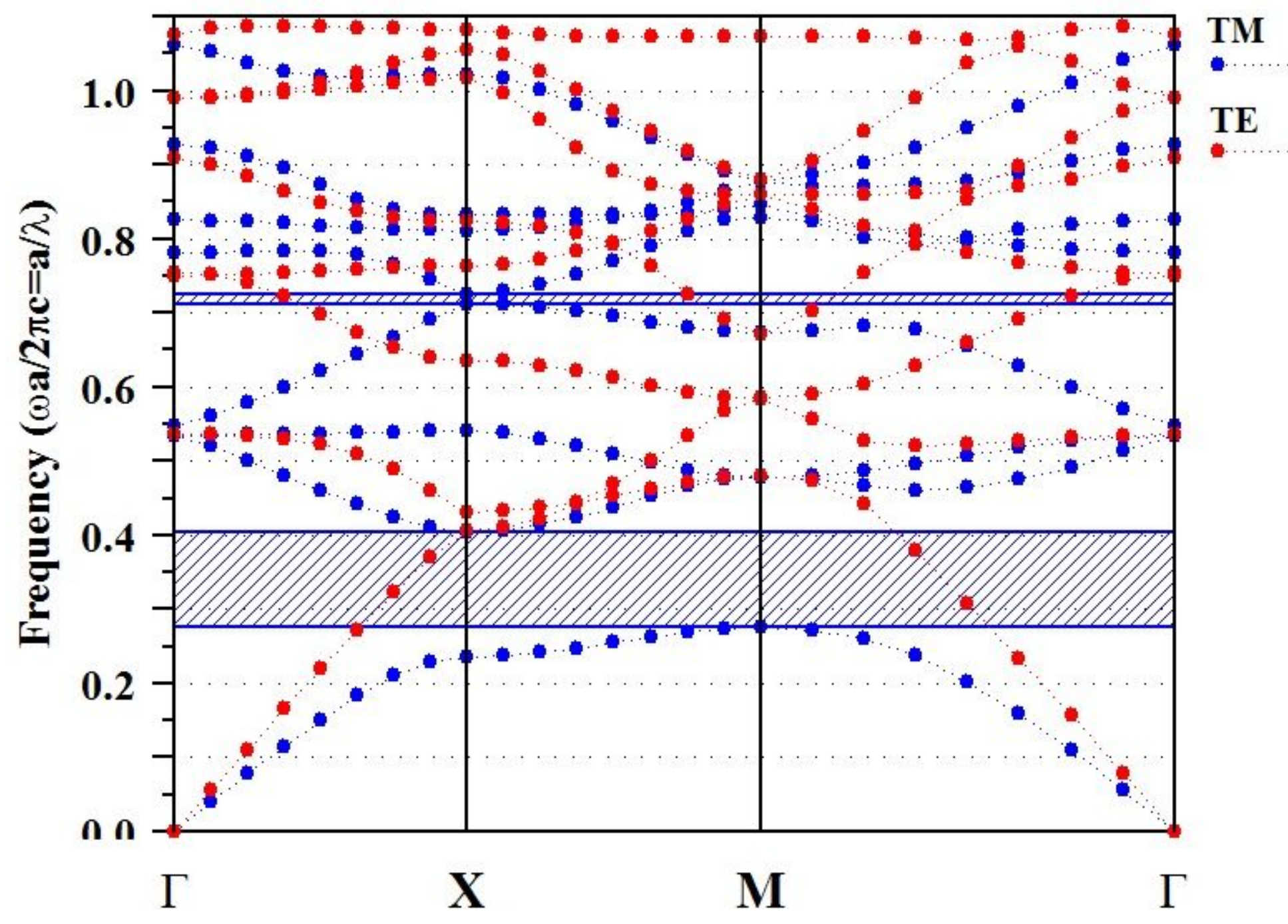

Figure 1

The band structure diagram of the proposed photonic crystal 


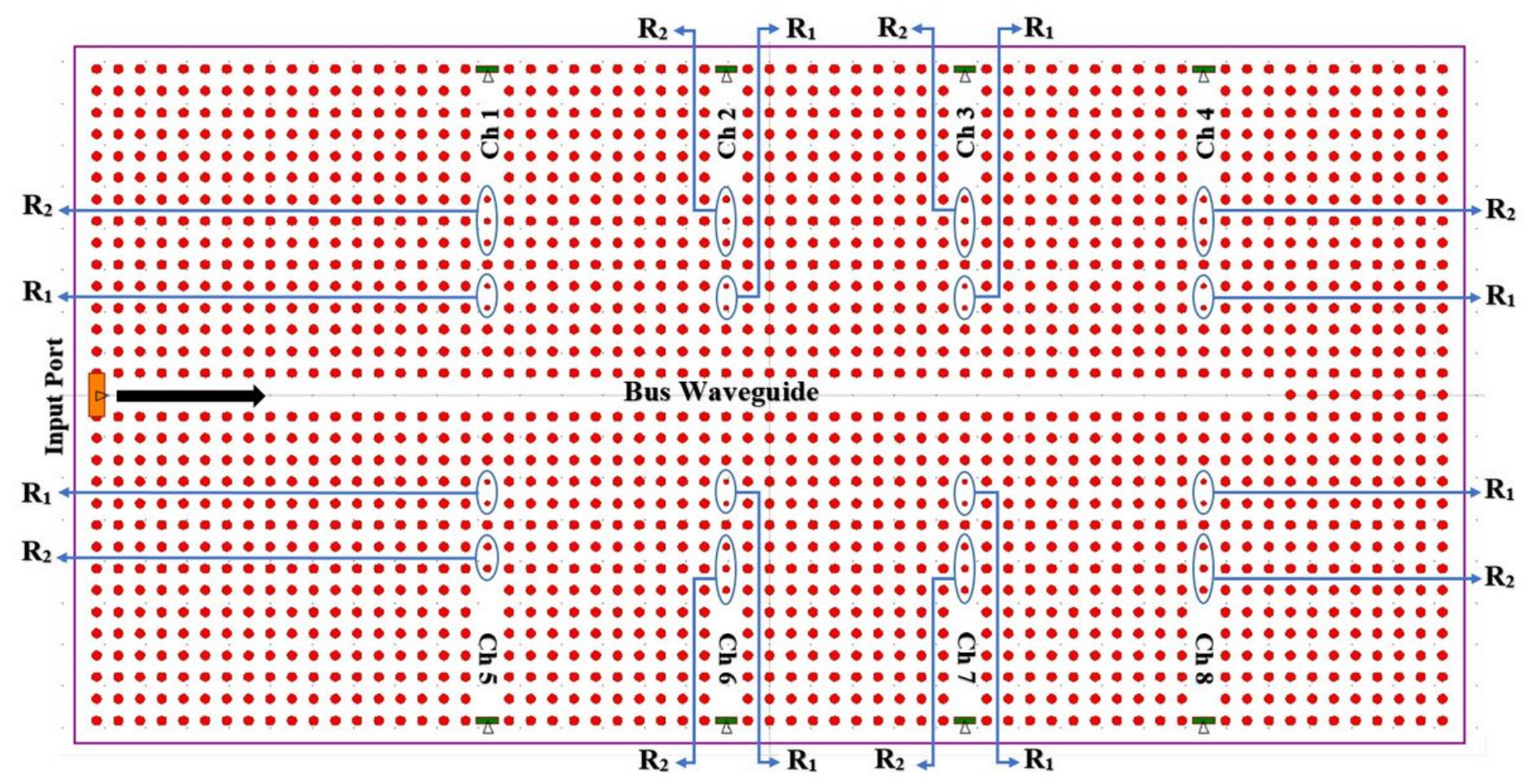

Figure 2

The schematic diagram of the proposed 8-channel PhC DEMUX 


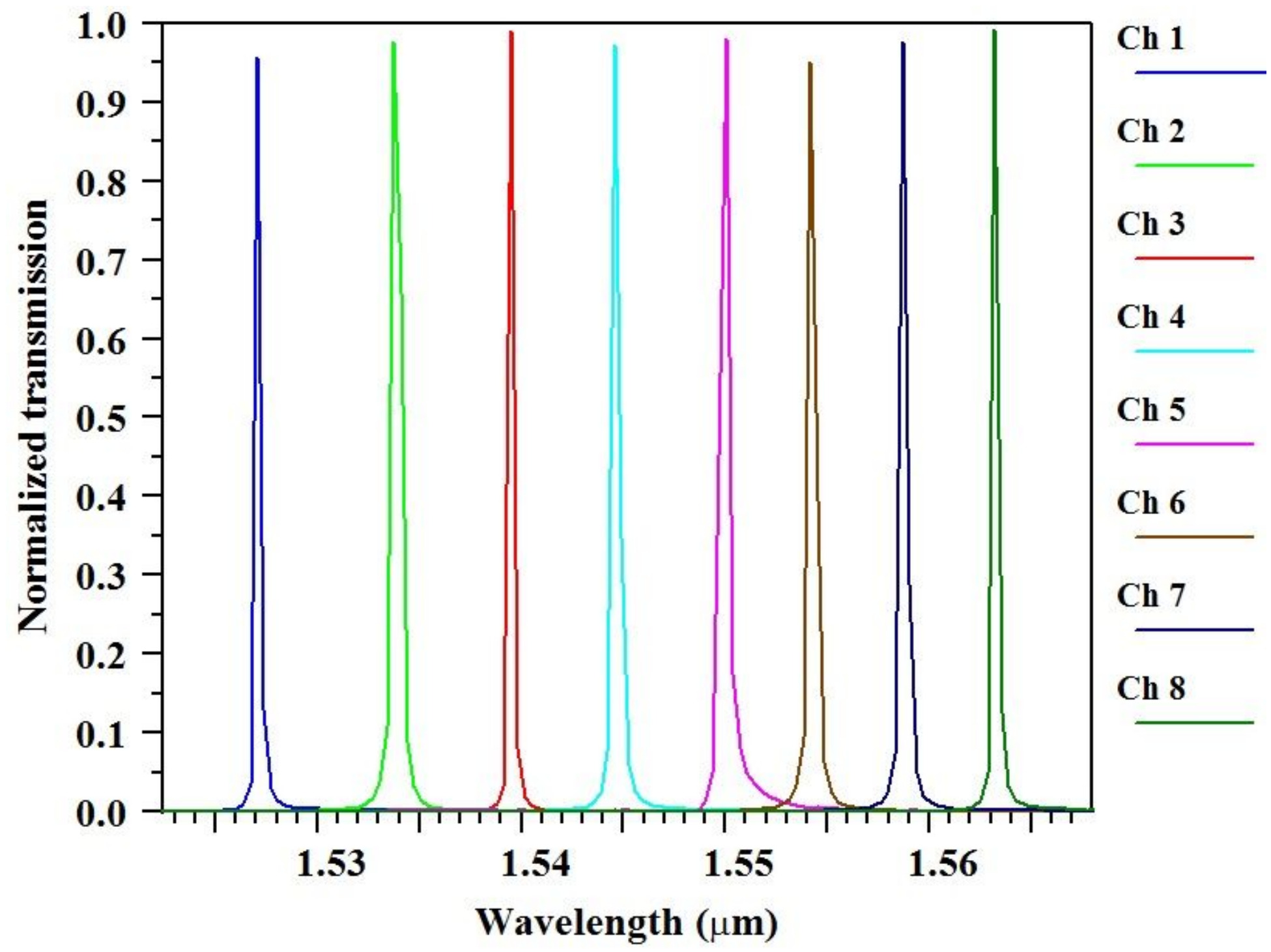

Figure 3

Output spectrum of the proposed 8-channel DEMUX 


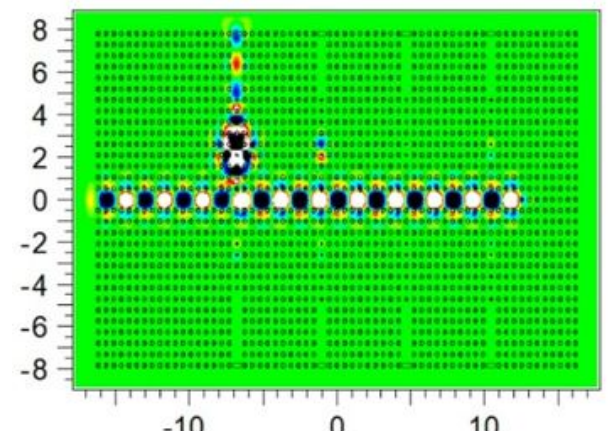

(a)

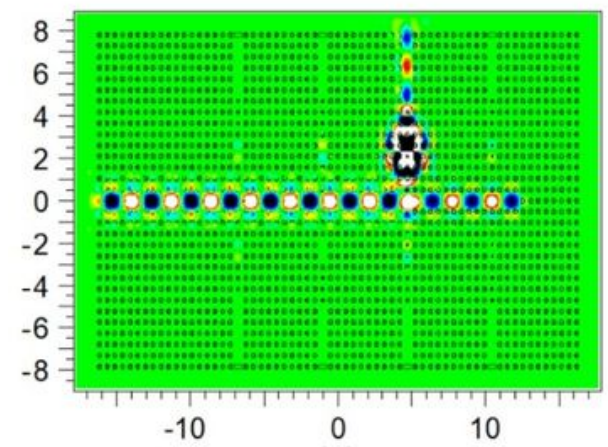

(c)

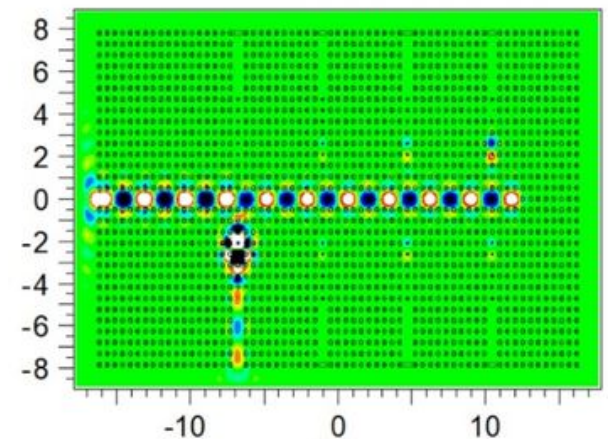

(e)

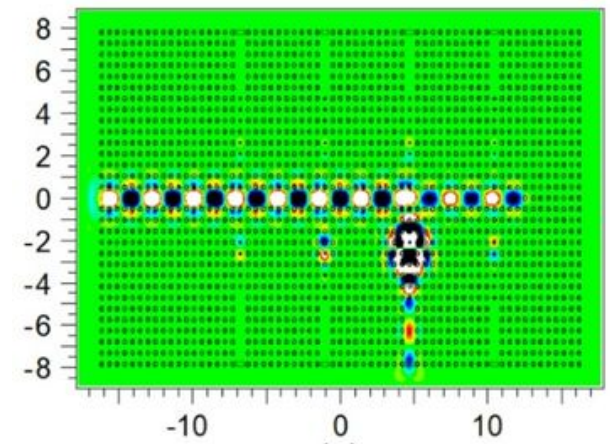

(g)

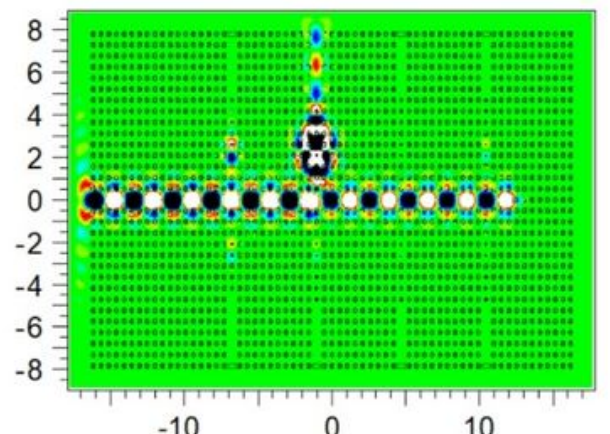

(b)

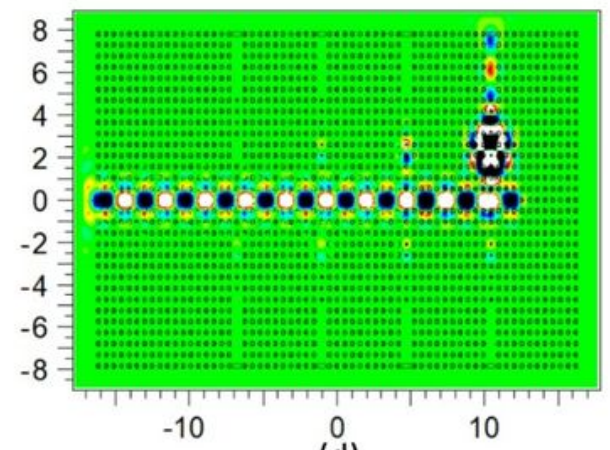

(d)

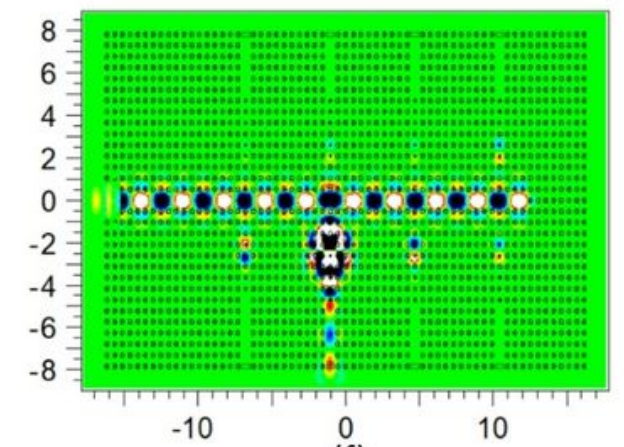

(f)

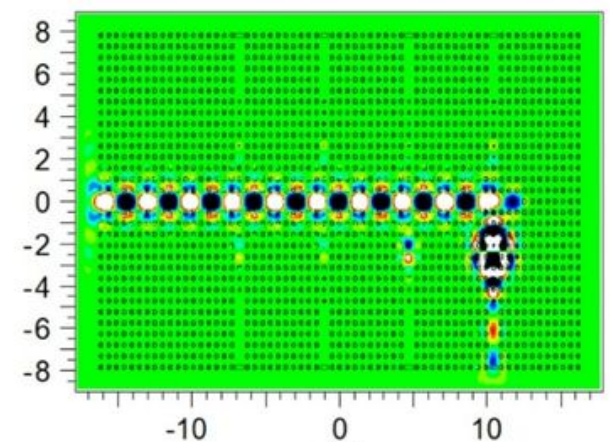

(h)

Figure 4

Electric field distribution of the proposed DEMUX in channels 1 (a), channel 2 (b), channel 3 (c), channel 4 (d), channel 5 (e), channel 6 (f), channel 7 (g) and channel 8 (h) 


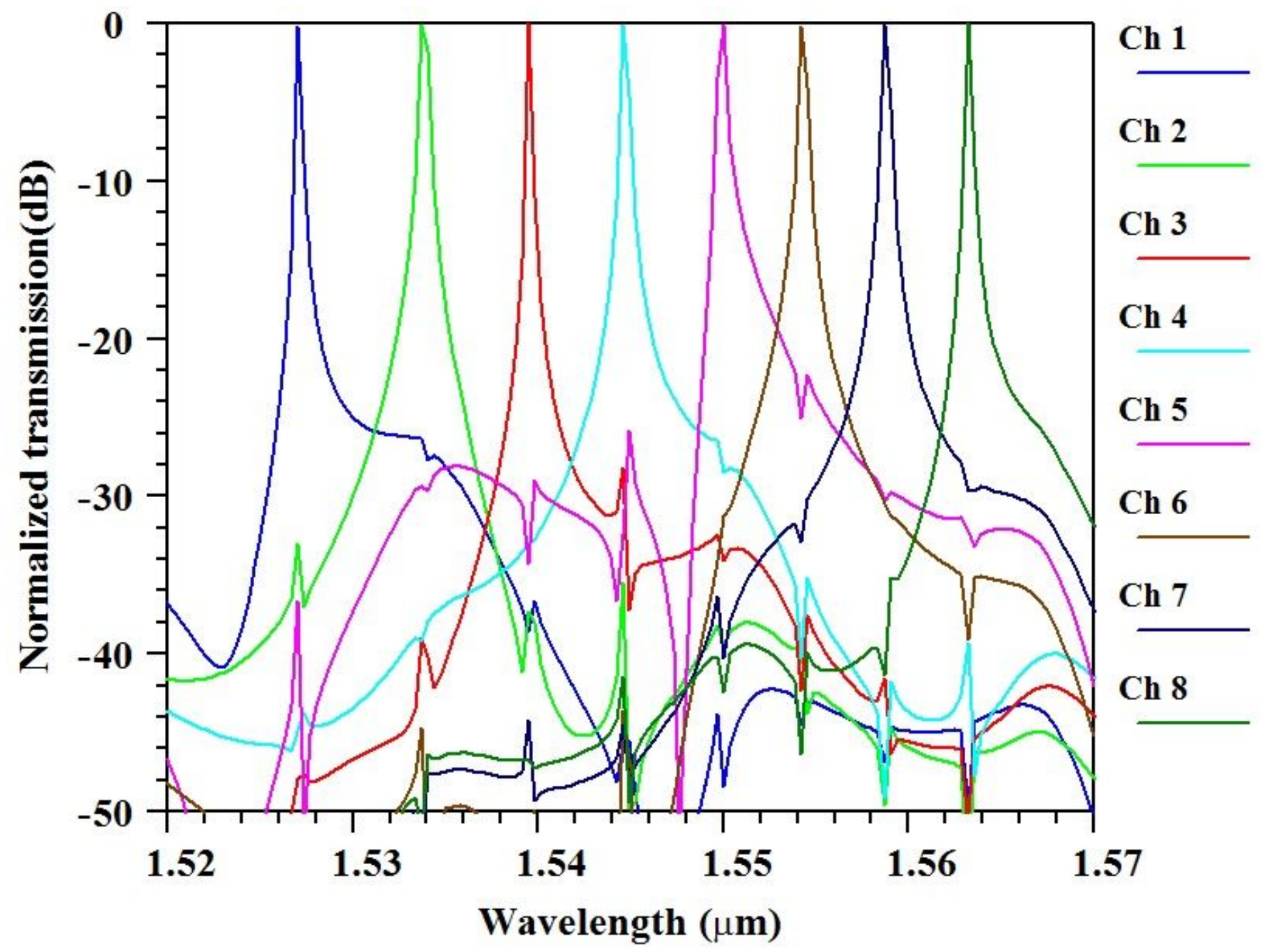

Figure 5

Output spectrum of the proposed 8-channel DEMUX (dB) 